\title{
Evaluation of neurological changes in secondary progressive multiple sclerosis patients treated with immune modulator MIS416: results from a feasibility study
}

Gill A. Webster ${ }^{1 *}$, Dalice A. Sim², Anne C. La Flamme ${ }^{3}$ and Nancy E. Mayo ${ }^{4}$

\begin{abstract}
Background: While disease progression can be readily monitored in early stage relapsing multiple sclerosis (MS), it is more challenging for secondary progressive multiple sclerosis (SPMS). This advanced stage of disease has distinct pathophysiology due to compartmentalization of neuroinflammatory activity within the central nervous system, resulting in increased incidence and severity of cognitive dysfunction. The shift in the dominant disease pathways is underscored by the failure of relapsing therapies to benefit SPMS patients, highlighting the need for novel treatment strategies and clinical trial endpoints that are well-aligned with potential benefits. The Expanded Disability Status Scale (EDSS) is widely used but is weighted towards ambulatory ability, lacking sensitivity to other aspects of neurological impairment experienced in more severely disabled SPMS patients, so may not effectively capture their clinical status. To investigate the feasibility of an alternative clinical trial endpoint model for a phase $2 \mathrm{~B}$ trial of an immune modulator for SPMS, the potential for treatment efficacy-based patient-centered outcomes was assessed within the context of a before and after, 12-week clinical trial of safety and tolerability.

Methods: Patients treated with MIS416 for 12 weeks were evaluated for clinical status at baseline and end of dosing, using the established Multiple Sclerosis Functional Composite, Short Form Health Survey, and Expanded Disability Status Scale. Responder status was determined for eight outcome measures based on minimally important change, defined using published studies. To evaluate the patients' immune response to MIS416, blood plasma samples collected at baseline and pre- and 24-h post doses 1-4 were analyzed using multiplex cytokine quantification assays.
\end{abstract}

Results: Using a combination of patient-centered outcomes, MIS416 treatment was associated with improved clinical status for 10/11 patients: eight patients showed improvement on two to five outcome measures, five of which also showed improvement by EDSS. Multi-dimensional scaling analysis of MIS416-induced factors quantified in individual patients, revealed immune response patterns which had a strong concordance with the extent of the patients' clinical response.

Conclusions: The data support the feasibility of using patient-centered outcomes as additional clinical trial endpoints, for determining the efficacy of disease-modifying therapies, in secondary progressive multiple sclerosis patients.

Trial registration: ClinicalTrial.gov, NCT01191996

Keywords: Secondary progressive multiple sclerosis, MIS416, Immune modulator, Myeloid cells, Neurological improvement, Performance related outcome, Patient-reported outcome, Plasma immune biomarker

\footnotetext{
* Correspondence: gill@innateimmuno.com

${ }^{1}$ Innate Immunotherapeutics Ltd, Auckland, New Zealand

Full list of author information is available at the end of the article
} 


\section{Background}

Multiple sclerosis (MS) is a chronic autoimmune disease of the central nervous system (CNS) [1], resulting in the occurrence of inflammatory lesions (plaques) anywhere within the white matter of the CNS, most commonly the periventricular regions, optic nerves, brain stem, cerebellum, and spinal cord [1, 2]. The symptoms and impairments experienced by people with MS vary and depend on the affected areas of the CNS [3, 4]. MS affects three times as many women as men and is typically diagnosed between the ages of 15 and 40 years [5]. It is estimated that there are 2.3 million individuals with MS worldwide [6]. The highest prevalence of MS is found in Canada, USA, and other northern European countries with rates ranging from 50 to 240 per 100,000 population [6, 7]. The most common type of MS is relapse-remitting MS (RRMS) [8], affecting 75-85\% of patients, and characterized by clear and well-defined relapses followed by complete or partial remissions [8]. About $50 \%$ of individuals diagnosed with RRMS will develop secondary progressive MS (SPMS) after 10-15 years [8]. SPMS is characterized by steady worsening of the condition, with or without attacks and remissions [8]. Primary progressive MS (PPMS) affects about $10 \%$ and is characterized by continuous and slow worsening of symptoms from the onset of the condition, with no clear relapses or remissions [8]. The least common and most severe type of MS is progressive-relapsing MS (PRMS), which occurs in about $5 \%$ of the persons diagnosed with MS [8]. This type is characterized by steady worsening of the condition with clear attacks and without remissions [8].

MIS416 is a myeloid-targeted immune response modifier currently in a phase $2 \mathrm{~B}$ randomized, double-blind, placebo-controlled trial to study the efficacy and safety in the treatment of subjects with secondary progressive multiple sclerosis (SPMS) (http://clinicalTrials.gov identifier NCT02228213). The data from a clinical trial to determine safety and tolerability has been previously published [9]. In contrast to relapse remitting MS (RRMS) therapies that target the peripheral "outside-in" adaptive immune biology, MIS416 has been developed to modulate the secondary progressive stage of MS, which is considered to reflect self-perpetuating innate inflammation that has become contained within the central nervous system (CNS) [10]. This shift in disease pathophysiology is emphasized by the failure of RRMS therapies to alter disease progression in SPMS patients [11]. Early pre-clinical and human compassionate use studies have demonstrated the ability of MIS416 to enhance several myeloid-directed anti-inflammatory pathways that can potentially access the CNS, leading to inhibition of this compartmentalized neuroinflammation and the promotion of endogenous $\mathrm{CNS}$ repair pathways $[12,13]$. Accordingly, to fully evaluate the clinical potential of MIS416, measures of CNS function that are sensitive to SPMS-associated chronic neuroinflammatory status are desirable.

Currently, the Expanded Disability Status Scale (EDSS) is the only regulatory-approved measure of change in disease activity and burden in MS patients [14]. However, reported limitations of the EDSS [14], such as lack of sensitivity to cognitive and arm function, mean that for patients with advanced disease, improvements in clinical status may not be captured by EDSS, which is biased towards the patient's walking ability [15]. Given the recognized limitations of the EDSS, there is increased focus on the refinement and validation of patient-reported outcomes (PROs) and performancerelated outcomes (PerfOs), to account for the full range of MS symptoms [16, 17]. This shifting ground with respect to the important outcomes of MS therapy and the need to include patient-centered outcomes [18] raises uncertainty about the feasibility of including outcomes beyond the EDSS in trials to determine the treatment effect of MIS416 in SPMS patients. A recent paper by the Multiple Sclerosis Outcome Assessments Consortium (MSOAC) [19] has outlined the need for better outcome measures for MS. In preparation for a shift in the recommended outcomes for MS, this study aimed to investigate the feasibility of an alternative clinical trial endpoint model. Accordingly, we conducted a preliminary analysis of PRO and PerfO secondary endpoint measures from the dose-confirmation phase of a trial on MIS416-treated SPMS patients, to provide data for assessment of response on the patient-centered outcomes. As peripheral blood immune biomarkers associated with MIS416 mechanism of action are known $[9,20]$, patient samples collected immediately prior to and $24 \mathrm{~h}$ after MIS416 administration, for doses 1-4, were analyzed to determine any relationship between the pattern of the patient's immune response to MIS416 and their change in clinical status, as indicated by these PerfOs and PROs. The overall aim was to support the feasibility of using patient-centered outcomes to measure response to MIS416 treatment in SPMS patients in a larger, placebocontrolled efficacy study.

\section{Methods}

\section{Clinical trial design}

A single-center, open-label, non-randomized, doseescalation study was conducted in two phases: a doseescalation (DE) phase, to evaluate the safety, tolerability, and maximum tolerated dose (MTD) of MIS416 administered intravenously, once weekly for four doses; and a dose-confirmation (DC) phase, comprising a single cohort treated at or below the MTD of MIS416, dosed once weekly for up to 12 doses [9]. The study was conducted in accordance with the Declaration of Helsinki 
[21] and was approved by the Upper South A Health and Disability Ethics Committee (URA/10/01/011). Written informed consent for blood sample collection and analysis was obtained from all study participants prior to the trial. The methods we are reporting follow the recommendations from the new CONSORT extension to randomized pilot and feasibility trials [22].

\section{Patients}

Study participants were recruited from Christchurch, New Zealand, where the clinical trial site was located. All patients provided informed consent prior to screening. Exclusion criteria included treatment with any immunomodulatory therapy in the previous 6 months or vaccine/corticosteroid in previous 60 days, as well as any diseases that might impact on the patients' diagnosis and evaluation of MS. Altogether, 34 patients (20 females) 18 years or older were enrolled, 19 in the DE and 15 in the DC phase. All had a diagnosis of MS based on McDonald's criteria [23], either primary or secondary progressive MS, evidence of worsening clinical status over the previous 2 years, and EDSS scores of 2.5-7.0 at screening. Enrollment in the DC phase was limited to patients with SPMS, to support a planned phase 2 trial in this more homogeneous population. The feasibility study to investigate patient-centered outcomes was conducted on the DC cohort only.

\section{Patient clinical status and measures}

The clinical status of patients who completed the DC phase (MIS416 weekly for 12 weeks) was assessed before and after completion of the study using the EDSS, Multiple Sclerosis Functional Composite (MSFC) [24], Fatigue Severity Scale (FSS) [25], and the Short Form Health Survey-36 (SF-36) [26].

\section{Calculation of change and responder status on performance-rated outcome measures and patient-reported outcome measures (patient-centered outcomes)}

Minimal important change (MIC) was used to define a positive patient responder status as estimated from published studies. For EDSS, MIC was 0.5 [27]. From the MFSC [24], MIC values were determined for the following performance-rated outcomes (PerfOs): gait speed (GS) $0.10 \mathrm{~m} / \mathrm{s}$ [28]; Paced Auditory Serial Addition Test (PASAT) 9 (based on changes greater than estimates of the practice effect) [29, 30]; Nine-Hole Peg Test (NHPT) $20 \%$ [29]. For the SF-36, four subscales that were most closely related to the biological action of the investigational drug were included as outcomes. These were physical function to reflect the everyday impact of improved gait speed or walking; mental health to reflect both a primary effect or a secondary effect from walking better; vitality for fatigue as it is the most distressing symptom of people with MS and not at all captured by the EDSS; and general health as improved walking, mood, and fatigue will impact this outcome and it is an important patient-centered outcome [31]. The other four subscales, pain and the three role subscales, were not included, as pain is often either neuropathic in origin or musculo-skeletal secondary to abnormal walking pattern, and the role variables are downstream outcomes from improved mobility and mental health.

For these subscales, a change in 10 points on a $0-100$ scale for each score was considered a MIC [32] (equivalent to $1 / 2$ standard deviation $(\mathrm{SD})$ as $\mathrm{SD}$ is approximately 20 in adults $>55$ years from a large nominative sample) [30]. The FSS was originally included in the test battery, but we did not want to include two PROs which both measured fatigue, and as the other PROs came from the SF-36, to add homogeneity, we included only SF-36 PROs. In addition, the items of the FSS $(n=9)$ do not measure severity of fatigue but rather causes, consequences, and impact on daily life.

Each patient was classified as a responder (1) or non-responder (0) on the EDSS and the three PerfOs and four PROs from the SF-36. Response across all measures was summarized using the total number of measures with an observed response. Response pattern was used to rank patients based on total number of responses with priority given to responses on PerfO. Patients were then classified into three groups based on the distribution of ranks: high, medium, and low responder.

\section{Quantification of plasma MIS416 immune biomarkers analysis}

For quantification of immune factors in patient plasma, heparin anti-coagulated peripheral blood was collected pre-treatment (baseline), as well as pre- and 24-h post MIS416 administration for doses 1-4. Following immediate processing of blood, plasma was isolated and stored at $-80{ }^{\circ} \mathrm{C}$ until analysis. The immune factors selected were based on their capacity to reflect different aspects of MIS416-mediated immune activation, as determined in a pilot study conducted on plasma from patients who completed the DE phase of the trial: type I/II interferon signaling (IP-10, MIG, MIP-1 $\alpha$, neopterin, IFN- $\gamma$ ); pro-inflammatory mediators (IL-6, IL-11, GCSF, IL-12p40, PGE2); cell migration (fractalkine, rantes, MCP-1); anti-inflammatory mediators (TGF- $\beta$, soluble TNFR1, IL-10, IL-1RA); cell adhesion (CD62E, ICAM1, VCAM1); and the growth factor, VEGF. The concentrations $(\mathrm{pg} / \mathrm{mL})$ of these cytokines and chemokines were determined using a custom cytokine bead array matrix (Becton Dickinson CBA Flex Sets ${ }^{\mathrm{TM}}$ ) or by ELISA (BD Biosciences) according to the manufacturer's instructions. 


\section{Statistical analysis}

\section{Patient-centered outcomes}

Each patient was classified as a responder or nonresponder on the EDSS and the three PerfOs and four PROs from the SF-36. Response across all measures was summarized using the total number of measures with an observed response. Participants were ranked in order of total responses across PerfOs and PROs. The probability of observing response patterns of the magnitude determined was estimated by referring to the binomial distribution and assuming a spontaneous response rate to be rare $(\leq 3 / 10)$, given the population under study.

\section{Immunological factors}

To evaluate the extent to which each patient's immune system was altered in response to MIS416 therapy, the maximum recorded plasma concentration for each immune factor across dose 1-4 time points was used for further analysis. The data were first normalized (subtracted the mean and divided by the standard deviation) so that all were equally weighted, then the resemblance between each pair of patients was determined. This was calculated as the sum (over all immune factors) of the squared differences between patients. Thus, for each pair $(i, j)$ of patients,

$$
\operatorname{Resemblance}(i, j)=\sum_{21}\left(V_{i}-V_{j}\right)^{2}
$$

where $V_{i}$ and $V_{j}$ are the values for patients $i$ and $j$ respectively. Once the resemblances were calculated, a cluster algorithm was used to generate a multidimensional scaling analysis plot to illustrate the differences between patients. SIMPER (similarity percentages) analysis was used to determine which immunological factors mostly accounted for the differences between patients $[33,34]$. These factors were used subsequently to compare the patient immune responses with the patientcentered clinical response.

\section{Statistical analysis of immune response biomarkers in patients grouped by clinical responder status}

Biomarker levels for patients grouped according to their clinical responder status (high, medium, or low) were compared using a two-way ANOVA followed by Holm-Sidak's multiple comparison post-test (PRISM software version 7.0; GraphPad). A value of $P<0.05$ was taken as significant.

\section{Results}

Of the targeted 15 patients enrolled in the DC phase, 11 subjects completed all 12 weekly doses. Two subjects withdrew for personal reasons and 2 subjects were withdrawn due to an adverse event which occurred early on in dosing schedule. Adverse events were headache $(n=2)$ and pain in extremity (in the arm or the leg) $(n=1)$.

The distribution of clinical status measures prior to MIS416 treatment of the patients who completed the DC phase [9] are summarized in Table 1.

\section{Patient change and responder status on EDSS, PerfOs, and PROs}

The amount of change on the EDSS and the three PerfO measures in absolute terms, or percent change from baseline for the NHPT, as well as how each patient was classified on responder status for each of the measures is shown in Table 2.

Five patients were responders on EDSS and 4 of those also responded on gait speed. There were 4 responders on PASAT and 0 on NHPT. In all, 7/11 patients were responders on at least one of these measures.

Table 3 shows the amount of change in absolute terms for the four PROs. Of these, there were 6 responders on vitality; 3,2 , and 1 patients responded on general health, mental health, and physical functioning respectively, with a total of $8 / 11$ patients responding on at least one PRO.

For EDSS/PerfOs the probability of observing 7/11 responders, even assuming a spontaneous response as high as $30 \%$ in the absence of intervention is 0.022 . The probability of observing 8/11 responders on PRO is 0.004 (http://stattrek.com/online-calculator/binomial.aspx).

Table 4 shows how each patient was classified as a responder ranked in order by total number of responding

Table 1 Distribution of the clinical characteristics of the patient sample group (DC cohort; $n=11$ ) for age, EDSS, PerfOs, and PROs prior to MIS416 treatment

\begin{tabular}{|c|c|c|c|}
\hline & Median & Minimum & Maximum \\
\hline Age (years) $(n=11)$ & 53 & 46 & 60 \\
\hline EDSS & 6 & 4 & 7 \\
\hline${ }^{\mathrm{a} G a i t}$ speed (m/s) & 0.68 & 0.26 & 1.19 \\
\hline NHPT (s) & 28.5 & 19.8 & 152.3 \\
\hline 'PASAT (errors) & 37 & 23 & 60 \\
\hline \multicolumn{4}{|l|}{${ }^{\mathrm{C} S F-36}[$ Norm]/100 } \\
\hline General health [75] & 46.1 & 23.7 & 65.1 \\
\hline Physical function [82] & 23.1 & 19.3 & 40.3 \\
\hline Bodily pain [75] & 42.6 & 30.6 & 62 \\
\hline Role physical [81] & 30.2 & 2.5 & 39.2 \\
\hline Role emotional [88] & 45.7 & 17.9 & 56.2 \\
\hline Social function [88] & 37.3 & 17.2 & 57.3 \\
\hline Vitality [68] & 40.7 & 25.9 & 52.6 \\
\hline Mental health [80] & 50.9 & 29.9 & 61.3 \\
\hline
\end{tabular}

${ }^{a} n=10$

${ }^{\mathrm{b}}$ Maximum score possible $=60$

${ }^{c}$ Higher $=$ better health 
Table 2 Change and responder status (RS) on EDSS and PerfOs

\begin{tabular}{|c|c|c|c|c|c|c|c|c|}
\hline \multirow[t]{2}{*}{ Patient ID } & \multicolumn{2}{|l|}{ EDSS } & \multicolumn{2}{|c|}{ Gait speed } & \multicolumn{2}{|l|}{ PASAT } & \multicolumn{2}{|l|}{ NHPT } \\
\hline & Change & RS & Change & RS & Change & RS & $\%$ change & RS \\
\hline DC01 & 3.5 & 1 & 0.10 & 1 & 9 & 1 & -3.48 & 0 \\
\hline DC02 & 0 & 0 & -0.05 & 0 & -14 & 0 & 1.91 & 0 \\
\hline DC03 & -0.5 & 0 & -0.17 & 0 & -2 & 0 & -4.81 & 0 \\
\hline DC05 & 1 & 1 & 0.10 & 1 & 0 & 0 & 1.40 & 0 \\
\hline DC06 & 0 & 0 & 0.035 & 0 & 1 & 0 & -7.30 & 0 \\
\hline DC07 & 0.5 & 1 & 0.14 & 1 & 6 & 0 & -5.03 & 0 \\
\hline DC09 & 0 & 0 & 0.07 & 0 & 15 & 1 & -1.44 & 0 \\
\hline DC10 & 0.5 & 1 & 0.14 & 1 & -4 & 0 & -10.26 & 0 \\
\hline $\mathrm{DC} 11^{\mathrm{a}}$ & 0 & 0 & . & . & 11 & 1 & -13.79 & 0 \\
\hline DC12 & 0.5 & 1 & -0.10 & 0 & 24 & 1 & 42.42 & 0 \\
\hline DC14 & 0 & 0 & 0.04 & 0 & 0 & 0 & 7.53 & 0 \\
\hline
\end{tabular}

${ }^{\mathrm{a} N o n-a m b u l a t o r y ~ p a t i e n t ~ h e n c e ~ n o ~ g a i t ~ s p e e d ~ m e a s u r e s ~}$

outcomes. While the ranking of patients was based on total number of responses, priority was given to responses on PerfO. All but one patient (DC14) showed a response on at least one variable. The patient ranking was further classified as high, medium, and low responder based on $\geq 3,2$, and $\leq 1$ total number of responses accordingly (Table 4).

\section{Concordance of clinical responder status ranking with MIS416 pharmacodynamic immune response}

MIS416 is composed of immune stimulatory ligands for innate receptors, toll-like receptor 9 (TLR-9), and nucleotide-binding oligomerization domain-containing protein 2 (NOD-2) [20]. As a result of immune crosstalk under the influence of TLR-9-dependant type I interferon signaling and NOD-2-dependant NFkB signaling, regulatory and anti-inflammatory immune activity can be established [12, 13, 35, 36]. Analysis of the patients' immune response demonstrated that immune proteins associated with these pathways including the regulatory immune factors IFN- $\gamma$ [37] and IL-10 [38] were transiently increased in patients' plasma following MIS416 administration (maximal responses measured for each patient/immune factor are summarized in Table 5).

The multidimensional analysis of the maximal response (normalized) for each immunological parameter is presented as a non-metric multidimensional scaling plot (Fig. 1)

The clustering pattern for the 11 patients based on similarities of their immune response pattern to MIS416 showed a major cluster defined by 5 patients, and a small cluster of 2 patients, with 4 patients showing no clustering. This grouping of patients based on their immune response demonstrated a high degree of overlap with the grouping of patients based on their clinical response ranking. High clinical responders were defined by having demonstrated at least three responses on PerfO and PRO, medium responders had two responses,

Table 3 Change and responder status (RS) on PROs

\begin{tabular}{|c|c|c|c|c|c|c|c|c|}
\hline \multirow[t]{2}{*}{ Patient ID } & \multicolumn{2}{|c|}{ Physical function } & \multicolumn{2}{|c|}{ General health } & \multicolumn{2}{|l|}{ Vitality } & \multicolumn{2}{|c|}{ Mental health } \\
\hline & Change & RS & Change & RS & Change & RS & $\%$ change & RS \\
\hline DC01 & 17.2 & 1 & 7.1 & 0 & 14.9 & 1 & 7.8 & 0 \\
\hline DC02 & 0 & 0 & 0 & 0 & 14.9 & 1 & 2.6 & 0 \\
\hline DC03 & 0 & 0 & 12.9 & 1 & -2.9 & 0 & 2.6 & 0 \\
\hline DC05 & 9.6 & 0 & -2.4 & 0 & 11.9 & 1 & 10.5 & 1 \\
\hline DC06 & 5.3 & 0 & -9.6 & 0 & 11.9 & 1 & 10.5 & 1 \\
\hline DC07 & 3.8 & 0 & -7.1 & 0 & -3 & 0 & -5.2 & 0 \\
\hline DC09 & 9.6 & 0 & 14.3 & 1 & 2.9 & 0 & 5.2 & 0 \\
\hline DC10 & -1.9 & 0 & 9.5 & 0 & 14.9 & 1 & 7.9 & 0 \\
\hline DC11 & 7.6 & 0 & 14.3 & 1 & 11.9 & 1 & 5.3 & 0 \\
\hline DC12 & 0 & 0 & 1.4 & 0 & -8.9 & 0 & -23.6 & 0 \\
\hline DC14 & 0 & 0 & -2.4 & 0 & 0 & 0 & -2.6 & 0 \\
\hline
\end{tabular}


Table 4 Number of responses on PerfOs and PROs, responder rank, and classification of responder status

\begin{tabular}{llllll}
\hline Patient ID & PerfO & PRO & Total & Rank & Classification \\
\hline DC01 & 3 & 2 & 5 & 1 & High \\
DC05 & 2 & 2 & 4 & 3 & High \\
DC10 & 2 & 1 & 3 & 4 & High \\
DC11 & 1 & 2 & 3 & 5.5 & High \\
DC12 & 2 & 0 & 2 & 5.5 & Medium \\
DC07 & 2 & 0 & 2 & 7 & Medium \\
DC09 & 1 & 1 & 2 & 8 & Medium \\
DC06 & 0 & 2 & 2 & 9.5 & Medium \\
DC02 & 0 & 1 & 1 & 9.5 & Low \\
DC03 & 0 & 1 & 0 & 11 & Low \\
DC14 & 0 & 0 & 1 & Low \\
\hline
\end{tabular}

and low responders had $0-1$ (Table 4). Out of the major immunological cluster comprising 5 patients, 3 of these were also the highest responders on patient-centered outcomes and the other 2 were medium responders. Of the small immunological cluster of 2 patients, both of these were the lowest responders on the patientcentered outcomes.

Based on SIMPER analysis of the maximum observed value of each immune factor, those which provided the most information in discriminating patient immune responses to MIS416 were IFN- $\gamma$, MCP-1, MIG, MIP- $1 \alpha$, IL-6, and IL-10. To further examine the nature of these differences, the pattern of induction of immune factors by MIS416 by the high, medium, and low clinical responder groups were compared (Fig. 2).

The maximum levels of all immune factors, apart from IL-10, were detected at 24-h post-dose 1, and the responses were attenuated following subsequent doses.

Table 5 Maximum value of immune factors measured in sequential peripheral blood plasma samples collected at 24-h and 7-day post doses 1, 2, 3, or 4

\begin{tabular}{|c|c|c|c|c|c|c|c|c|c|c|c|}
\hline \multirow{3}{*}{$\begin{array}{l}\text { Immune } \\
\text { factor (pg/mL) }\end{array}$} & \multicolumn{11}{|c|}{ Patient ID } \\
\hline & DC & $D C$ & $D C$ & DC & $D C$ & DC & $D C$ & $D C$ & DC & DC & $D C$ \\
\hline & 1 & 2 & 3 & 5 & 6 & 7 & 9 & 10 & 11 & 12 & 14 \\
\hline CD62E & 18,503 & 33,350 & 16,905 & 21,510 & 18,458 & 37,502 & 19,729 & 20,984 & 27,616 & 18,471 & 8447 \\
\hline Fractalkine & 531 & 907 & 421 & 458 & 434 & 350 & 492 & 308 & 397 & 143 & 373 \\
\hline GCSF & 28 & 24 & 29 & 25 & 26 & 30 & 24 & 27 & 25 & 21 & 26 \\
\hline ICAM1 x104 & 70 & 74 & 51 & 38 & 64 & 145 & 59 & 48 & 66 & 21 & 40 \\
\hline IFN- $\gamma$ & 178 & 281 & 147 & 74 & 73 & 192 & 76 & 48 & 52 & 0 & 122 \\
\hline IL-10 & 11 & 19 & 12 & 8 & 7 & 27 & 10 & 7 & 11 & 5 & 29 \\
\hline IL11 & 322 & 423 & 2540 & 3194 & 19 & 0 & 179 & 808 & 168 & 1522 & 60 \\
\hline IL12p40 & 339 & 321 & 182 & 291 & 292 & 185 & 60 & 183 & 65 & 136 & 177 \\
\hline IL1RA & 6693 & 7696 & 5797 & 6315 & 3939 & 18,299 & 18,709 & 8070 & 6858 & 2721 & 4965 \\
\hline IL6 & 36 & 47 & 181 & 22 & 32 & 87 & 81 & 19 & 23 & 11 & 140 \\
\hline IP10 & 3640 & 5371 & 6089 & 2812 & 3068 & 7689 & 4240 & 1646 & 2704 & 1234 & 1395 \\
\hline MCP-1 & 163 & 722 & 889 & 432 & 330 & 296 & 385 & 187 & 255 & 704 & 465 \\
\hline MIG & 9766 & 111,584 & 13,706 & 24,823 & 5444 & 18,058 & 16,966 & 7520 & 2780 & 1514 & 2989 \\
\hline MIP1-a & 4 & 9 & 6 & 5 & 8 & 4 & 5 & 5 & 5 & 6 & 9 \\
\hline Neopterin & 5237 & 3378 & 3486 & 2244 & 4221 & 3927 & 3850 & 3728 & 3891 & 2060 & 2658 \\
\hline PGE2 & 182 & 219 & 157 & 160 & 192 & 212 & 343 & 398 & 296 & 207 & 32 \\
\hline RANTES & 255,145 & 174,684 & 189,866 & 231,938 & 166,300 & 257,508 & 353,761 & 229,399 & 265,178 & 167,513 & 119,622 \\
\hline TGF- $\beta$ & 6034 & 5973 & 3394 & 3872 & 4699 & 6542 & 6291 & 11,851 & 8124 & 8236 & 5047 \\
\hline TNF-R & 4850 & 5230 & 5060 & 4560 & 7870 & 3680 & 2970 & 2450 & 1930 & 5210 & 2510 \\
\hline VCAM-1 & 972,534 & 761,399 & 970,295 & $1,047,583$ & $1,300,968$ & 889,864 & 672,495 & $1,056,576$ & 467,492 & 936,152 & 828,607 \\
\hline VEGF & 116 & 67 & 28 & 37 & 76 & 45 & 144 & 88 & 80 & 81 & 9 \\
\hline
\end{tabular}




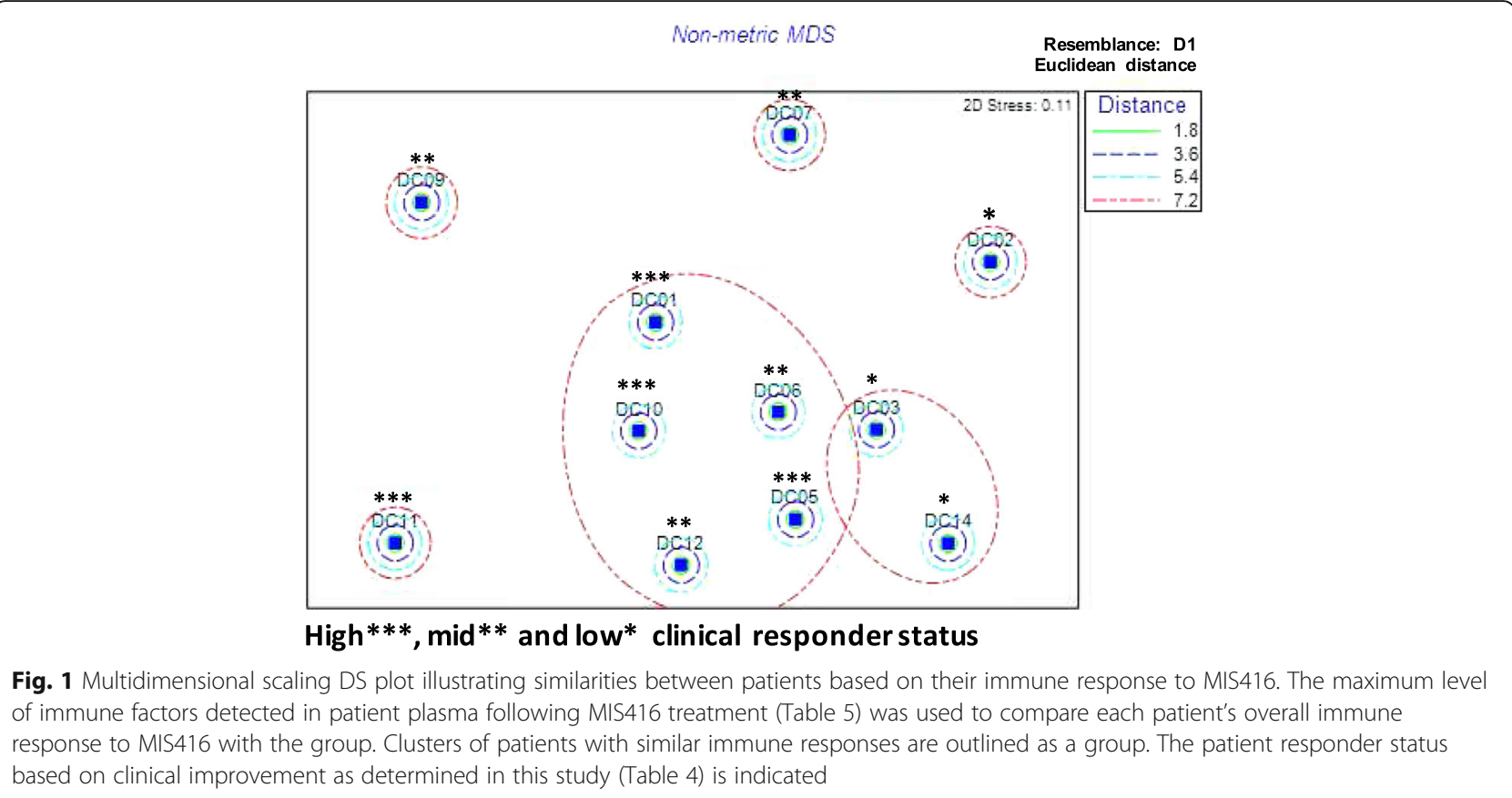

While this pharmacodynamic pattern of response was consistent for all patients, there were clear quantitative differences in the immune response to MIS416. Notably, low clinical responders had higher levels of IFN- $\gamma$ and IFN-inducible proteins MCP-1, MIG, and MIP- $1 \alpha$ than patients that were classified as high clinical responders. Furthermore, there were also higher levels of the NFkBdependant cytokines IL-6 and IL-10 [38, 39] in patients that were classified as low responders. Patients classified as medium responders aligned more closely with either the high- or the low-responder groups or for IL-6 and IL-10, they were midway between the two.

\section{Discussion}

\section{Clinical-patient-reported outcomes}

Of the 11 patients assessed here, 5 were classified as responders on the EDSS and of these, 4 were classified concordantly on gait speed, 2 on the PASAT, with no responders determined based on the NHPT. On any one of these 4 PerfOs, 7 people would have been classified as responders with 2 people showing a response uniquely on PASAT. For the PROs, only 1 of the EDSS responders showed a response on physical function (SF$36 \mathrm{PF}$ ) and this was the one person with a large EDSS response (DC01: EDSS response + 3.5). This may indicate only large changes on the EDSS will translate to a meaningful improvement in function from the patient's perspective. Other EDSS responders also showed response on vitality $(n=3)$ and mental health $(n=1)$. However, 5 people who were not EDSS responders showed response on one or more of the PROs. In an unblinded trial, PROs may change because patients wish to please the investigators and report more favorably on these outcomes. However, only four of the patients (DC02, DC03, DC06, DC11) did the response prevalence on PROs exceed that of the PerfOs suggesting that selfreport bias may not have been responsible for the response pattern observed here. PROs are also affected by a phenomenon called response shift [18], in which patients recalibrate their responses over time based on a change in perspective. Again, the concordance between the PerfO and PRO response, or the more frequent PerfO response $(n=7)$, does not support recalibration as the sole mechanism affecting the PRO response, suggesting that true change occurred.

\section{Patient immune response}

The observation that repeated dosing of MIS416 resulted in lower concentrations of biomarkers than were determined after dose 1 reflects desensitization of MIS416stimulated immune pathways. Typically, this pattern of response is associated with repeat exposure to therapeutics or ligands that engage IFN and NFKB signaling and their respective negative feedback pathways [35, 40-42]. This intrinsic regulatory mechanism is geared to control the host inflammatory response and is central to the maintenance of immune and tissue homeostasis [43]. That MIS416 showed activation of negative feedback pathways in patients is further evidence that NOD-2 and TLR-9 pathway activation by MIS416 occurred within the limits of immune homeostasis, which is important from both a safety and therapeutic standpoint. 

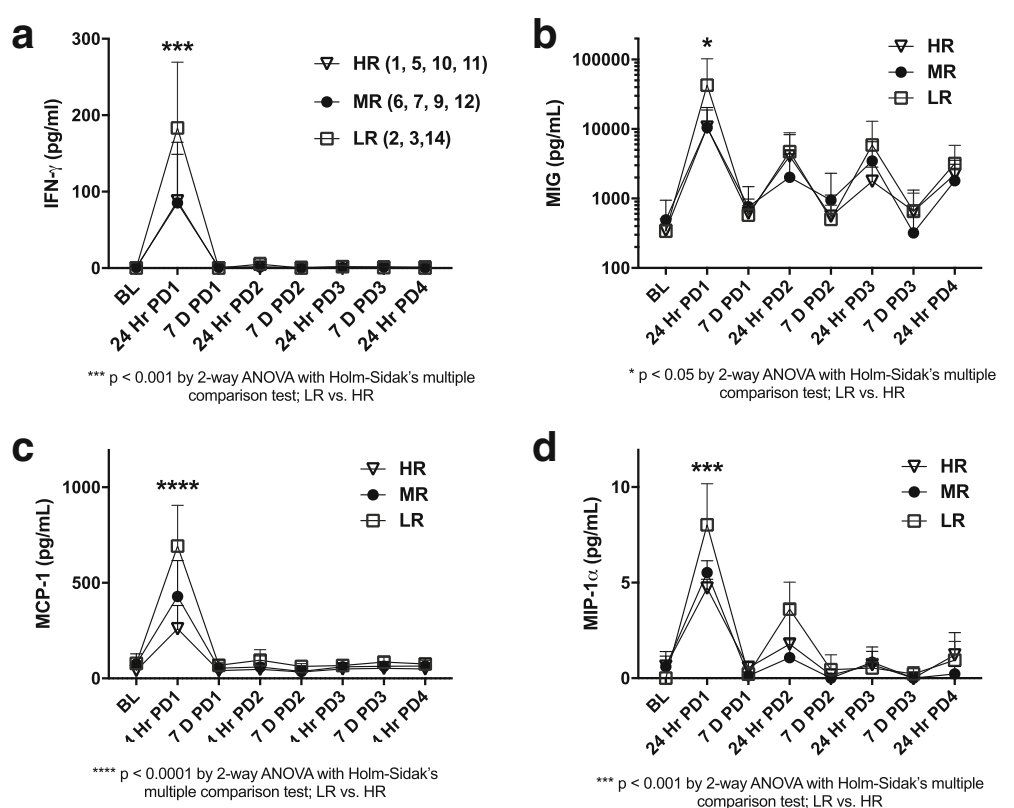

d
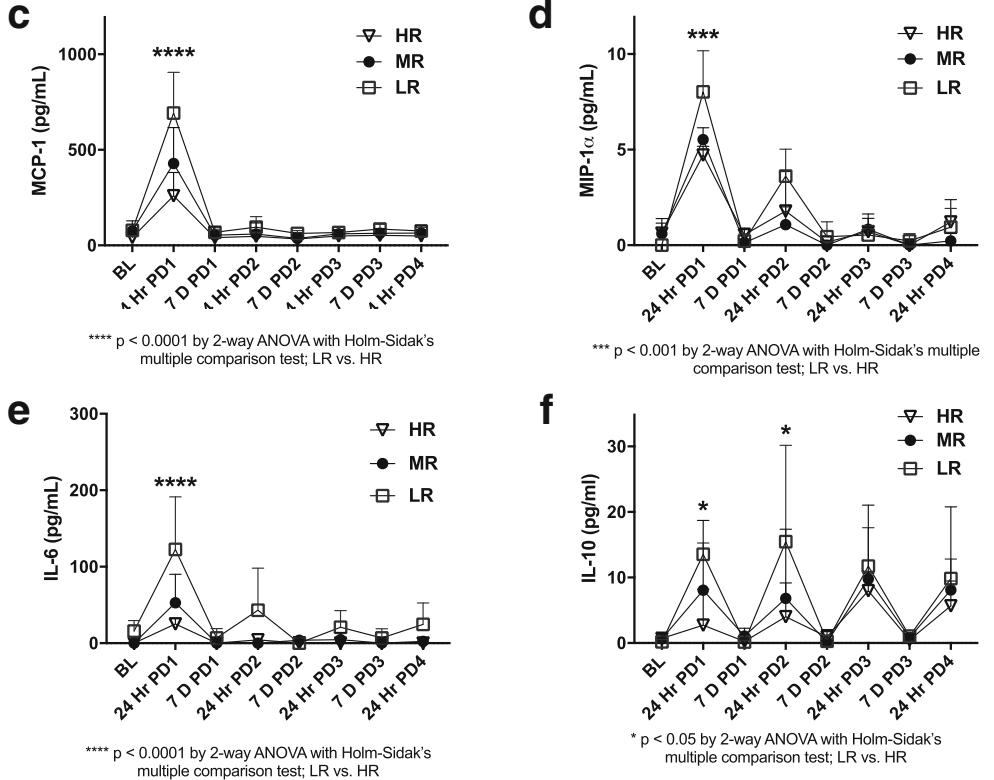

Fig. 2 MIS416 pharmacodynamic immune response of patients grouped according to change in clinical status. Immune proteins most important for discriminating differential immune responses to MIS416: (IFN-y (a), MIG (b), MCP-1 (c), MIP-1a (d), IL-6 (e), and IL-10 (f)) were quantified at 24hour (24 Hr) and 7 days (7 D) post doses (PD) 1-4 and values were compared between the patient groups defined as high responder (HR), medium responder (MR) or low responder (LR) based on their extent of clinical response. The patient ID numbers comprising each responder group are shown in parenthesis. Data shown are the mean values $(\mathrm{pg} / \mathrm{mL}) \pm S \mathrm{~S}$. Statistical significance was determined by two-way ANOVA followed by Holm-Sidak's multiple comparison: ${ }^{*} p<0.05 ; * * 0.005$; ${ }^{* * *} p<0.0001$

Immune-clinical relationship-patient-reported outcomes Analysis of the immunological response of the patients to MIS416 therapy supported their ranking based on the extent of their clinical improvement. While all patients responded immunologically in the same manner to MIS416, the patients that showed less clinical improvement had higher levels of MIS416 plasma biomarkers than those who were ranked as high responders. In particular, there were significantly more NFKB-dependant cytokines produced in these patients, although the significance ( $p$ value) of these differences should be treated with caution due to the small sample size. Such MIS416 hyper-responsiveness may be due to $\mathrm{NF}_{k} \mathrm{~B}$ gene and pathway mutations described in MS patients which are associated with higher constitutive NFKB activity and greater sensitivity to NFKB-activating agents $[44,45]$.

\section{Conclusions}

This study was limited by the low number of patients as well as the lack of a placebo control group. Notwithstanding these limitations, the results support the feasibility of pursuing these associations in a larger, placebo-controlled trial of MIS416 in SPMS patients and the evaluation of clinical status using a wider portfolio of patient-centered outcomes. Furthermore, in the context of such a study, concomitant analysis of the patient's immune response to MIS416 may provide additional insight into the significance of any change in clinical status measures, in particular those which are sensitive to change in inflammatory activity within the CNS.

\section{Abbreviations}

CD62E: E selectin; CNS: Central nervous system; DC: Dose confirmation; DE: Dose escalation; EDSS: Expanded Disability Status Scale; ELISA: Enzymelinked immunosorbent assay; FSS: Fatigue Severity Scale; GCSF: Granulocyte 
colony-stimulating factor; ICAM1: Intracellular adhesion molecule 1; IFNY: Interferon gamma; IL: Interleukin; IL-1RA: Interleukin 1 receptor antagonist; IP-10: IFN- $\gamma$-inducible protein of $10 \mathrm{kDa}$; MCP-1: Monocyte chemotactic protein-1; MIC: Minimal important change; MIG: Monokine induced by $\mathrm{Y}$ interferon; MIP-1a: Macrophage inflammatory protein; MSFC: Multiple Sclerosis Functional Composite; MTD: Maximum tolerated dose; NFkB: Nuclear factor kappaB; NHPT: Nine-Hole Peg Test; NOD-2: Nucleotide-binding oligomerization domain-containing protein 2; PASAT: Paced Auditory Serial Addition Test; PerfO: Performance-related outcome; PGE2: Prostaglandin E2; PRO: Patientreported outcome; RRMS: Relapse-remitting multiple sclerosis; SF-36: Short Form Health Survey-36; soluble TNFR1: Soluble tumor necrosis factor receptors 1; SPMS: Secondary progressive multiple sclerosis; TGF- $\beta$ : Transforming growth factor beta; TLR-9: Toll-like receptor 9; VCAM1: Vascular cell adhesion molecule 1; VEGF: Vascular endothelial growth factor

\section{Acknowledgements}

The authors gratefully acknowledge Rebecca Girvan and Victoria Pearson (Innate Immunotherapeutics), for technical assistance with the analysis of human plasma pharmacodynamics immune markers.

\section{Funding}

Innate Immunotherapeutics funded the clinical trial, collection of data, and laboratory determinations.

\section{Availability of data and materials}

By reasonable request, the quantitative datasets analyzed in this study are available from the corresponding author.

\section{Authors' contributions}

GW conceived the idea for the feasibility study, provided the raw data, worked with the other three authors to integrate the individual data sets meaningfully, and wrote the manuscript. NM developed and implemented the method for the pilot analysis of patient clinical status data, interpreted these results, and contributed to manuscript writing. DS was responsible for developing and implementing the method for analysis of the patients' immune response. ALF worked with DS on the analysis and interpretation of data that related to the patient's immune response. All authors read and approved the submitted manuscript.

\section{Ethics approval and consent to participate}

The study was conducted in New Zealand and was approved by the Uppe South A Health and Disability Ethics Committee (URA/10/01/011). Written informed consent for blood sample collection, analysis, and disclosure of anonymized data was obtained from all study participants prior to the trial.

\section{Consent for publication}

Not applicable.

\section{Competing interests}

Gill A Webster is an employee of Innate Immunotherapeutics. All other authors received no financial support in relation to their contribution to this work and have no financial relationship with any other organization or individuals that may have an interest in this work.

\section{Publisher's Note}

Springer Nature remains neutral with regard to jurisdictional claims in published maps and institutional affiliations.

\section{Author details \\ ${ }^{1}$ Innate Immunotherapeutics Ltd, Auckland, New Zealand. ${ }^{2}$ University of Otago, Wellington, New Zealand. ${ }^{3}$ Victoria University, Wellington, New Zealand. ${ }^{4}$ McGill University, Montreal, Canada.}

Received: 10 May 2017 Accepted: 27 October 2017

Published online: 16 November 2017

\section{References}

1. Noseworthy JH, Lucchinetti C, Rodriguez M, Weinshenker BG. Multiple sclerosis. N Engl J Med. 2000;343(13):938-52

2. Wingerchuk DM, Lucchinetti CF, Noseworthy JH. Multiple sclerosis: current pathophysiological concepts. Lab Investig. 2001;81(3):263-81.
3. Poser CM, Brinar W. Diagnostic criteria for multiple sclerosis. Clin Neurol Neurosurg. 2001;103(1):1-11.

4. Perkin GD. Mosby's Color Atlas and Text of Neurology, 2nd edn. London: Mosby; 2002.

5. Sadovnick AD. Genetic background of multiple sclerosis. Autoimmun Rev. 2012;11(3):163-6.

6. Rosati G. The prevalence of multiple sclerosis in the world: an update. Neurol Sci. 2001;22(2):117-39.

7. Alonso A, Hernán MA. Temporal trends in the incidence of multiple sclerosis: a systematic review. Neurology. 2008;71(2):129-35.

8. Lublin FD, Reingold SC. Defining the clinical course of multiple sclerosis: results of an international survey. National Multiple Sclerosis Society (USA) Advisory Committee on Clinical Trials of New Agents in Multiple Sclerosis. Neurology. 1996;46(4):907-11.

9. Luckey AM, Anderson T, Silverman MH, Webster G. Safety, tolerability and pharmacodynamics of a novel immunomodulator, MIS416, in patients with chronic progressive multiple sclerosis. Mult Scler J Exp Transl Clin. 2015;1:1-12.

10. Stys PK, Zamponi GW, van Minnen J, Geurts JJ. Will the real multiple sclerosis please stand up? Nat Rev Neurosci. 2012;13(7):507-14.

11. Shirani A, Okuda DT, Stüve O. Therapeutic advances and future prospects in progressive forms of multiple sclerosis. Neurotherapeutics. 2016;13(1):58-69.

12. White M, Webster G, O'Sullivan D, Stone S, La Flamme AC. Targeting innate receptors with MIS416 reshapes Th responses and suppresses CNS disease in a mouse model of multiple sclerosis. PLoS One. 2014;9(1):e87712.

13. White MP: Innate immunomodulation with MIS416: mechanism of action in experimental autoimmune encephalomyelitis. 2015.

14. Meyer-Moock S, Feng Y-S, Maeurer M, Dippel F-W, Kohlmann T. Systematic literature review and validity evaluation of the expanded disability status scale (EDSS) and the multiple sclerosis functional composite (MSFC) in patients with multiple sclerosis. BMC Neurol. 2014;14:58.

15. Polman $\mathrm{CH}$, Rudick RA: The multiple sclerosis functional composite: a clinically meaningful measure of disability. (1526-632X (Electronic)).

16. Schaffler N, Schonberg P, Stephan J, Stellmann JP, Gold SM, Heesen C Comparison of patient-reported outcome measures in multiple sclerosis. Acta Neurol Scand. 2013;128(2):114-21.

17. Bin Sawad A, Seoane-Vazquez E, Rodriquez-Monguio R, Turkistani F. Evaluation of the expanded disability status scale and the multiple sclerosis functional composite as clinical endpoints in multiple sclerosis clinical trials: quantitative meta-analyses. Curr Med Res Opin. 2016;32(12):1-6.

18. International Society of Quality of Life Research. Dictionary of the quality of life and health outcome measurement. In: Mayo NE, editor. Dictionary of the quality of life and health outcome measurement. 1st ed. Milwaukee: International Society of Quality of Life Research; 2015.

19. LaRocca NG, Hudson LD, Rudick R, Amtmann D, Balcer L, Benedict R, Bermel R, Chang I, Chiaravalloti ND, Chin P et al: The MSOAC approach to developing performance outcomes to measure and monitor multiple sclerosis disability. Mult Scler J. 2017:1352458517723718.

20. Girvan RC, Knight DA, O'Loughlin CJ, Hayman CM, Hermans IF, Webster GA. MIS416, a non-toxic microparticle adjuvant derived from Propionibacterium acnes comprising immunostimulatory muramyl dipeptide and bacterial DNA promotes cross-priming and Th1 immunity. Vaccine. 2011;29(3):545-57.

21. World Medical Association. World Medical Association Declaration of Helsinki: ethical principles for medical research involving human subjects. JAMA. 2013;310(20):2191-2194.

22. Eldridge $\mathrm{SM}$, Chan $\mathrm{CL}$, Campbell MJ, Bond CM, Hopewell S, Thabane L, Lancaster GA. CONSORT 2010 statement: extension to randomised pilot and feasibility trials. BMJ. 2016:355:i5239.

23. McDonald WI, Compston A, Edan G, Goodkin D, Hartung HP, Lublin FD, McFarland HF, Paty DW, Polman CH, Reingold SC, et al. Recommended diagnostic criteria for multiple sclerosis: guidelines from the international panel on the diagnosis of multiple sclerosis. Ann Neurol. 2001;50(1):121-7.

24. Fischer JS, Rudick RA, Cutter GR, Reingold SC. The Multiple Sclerosis Functional Composite Measure (MSFC): an integrated approach to MS clinical outcome assessment. National MS Society Clinical Outcomes Assessment Task Force. Mult Scler. 1999;5(4):244-50.

25. Lerdal A. Fatigue severity scale. In: Michalos AC, editor. Encyclopedia of quality of life and well-being research. Dordrecht: Springer Netherlands; 2014. p. 2218-21.

26. Ware JE Jr, Gandek B. Overview of the SF-36 Health Survey and the International Quality of Life Assessment (IQOLA) Project. J Clin Epidemiol. 1998;51(11):903-12. 
27. Kragt JJ, Nielsen IM, van der Linden FA, Uitdehaag BM, Polman CH. How similar are commonly combined criteria for EDSS progression in multiple sclerosis? Mult Scler. 2006;12(6):782-6.

28. Bohannon RW, Glenney SS. Minimal clinically important difference for change in comfortable gait speed of adults with pathology: a systematic review. J Eval Clin Pract. 2014;20(4):295-300.

29. Lamers I, Kelchtermans S, Baert I, Feys P. Upper limb assessment in multiple sclerosis: a systematic review of outcome measures and their psychometric properties. Arch Phys Med Rehabil. 2014;95(6):1184-200.

30. Norman GR, Sloan JA, Wyrwich KW. Interpretation of changes in health-related quality of life: the remarkable universality of half a standard deviation. Med Care. 2003;41(5):582-92.

31. DeSalvo KB, Bloser N, Reynolds K, He J, Muntner P. Mortality prediction with a single general self-rated health question: a meta-analysis. J Gen Intern Med. 2006;21(3):267-75.

32. Hopman WM, Towheed T, Anastassiades T, Tenenhouse A, Poliquin S, Berger C, Joseph L, Brown JP, Murray TM, Adachi JD, et al. Canadian normative data for the SF-36 health survey. Canadian Multicentre Osteoporosis Study Research Group. CMAJ. 2000;163(3):265-71.

33. Clarke K, Gorley, RN, 2015. PRIMER v7: User Manual/Tutorial. PRIMER-E, Plymouth, 296pp.: Clarke, KR, Gorley, RN, 2015. PRIMER v7: User Manual/ Tutorial. PRIMER-E, Plymouth, 296pp. Clarke, KR, Gorley, RN,2015 PRIMER v7: User Manual/Tutorial PRIMER-E, Plymouth, 296pp.

34. Clarke KR. Non-parametric multivariate analyses of changes in community structure. Aust J Ecol. 1993;18(1):117-43.

35. Hedl M, Abraham C. Secretory mediators regulate Nod2-induced tolerance in human macrophages. Gastroenterology. 2011;140(1):231-41.

36. Jha S, Pan-Yun Ting J. Holding the inflammatory system in check: NLRs keep it cool. F1000Prime Rep. 2015;7:15.

37. Kovarik P, Sauer I, Schaljo B. Molecular mechanisms of the anti-inflammatory functions of interferons. Immunobiology. 2007;212(0):895-901.

38. Saraiva M, O'Garra A. The regulation of IL-10 production by immune cells. Nat Rev Immunol. 2010;10(3):170-81.

39. Libermann TA, Baltimore D. Activation of interleukin- 6 gene expression through the NF-kappa B transcription factor. Mol Cell Biol. 1990;10(5):2327-34.

40. Makowska Z, Duong FHT, Trincucci G, Tough DF, Heim MH. Interferon- $\beta$ and interferon- $\lambda$ signaling is not affected by interferon-induced refractoriness to interferon-a in vivo. Hepatology. 2011;53(4):1171-80.

41. Kellogg RA, Tian C, Etzrodt M, Tay S. Cellular decision making by non-integrative processing of TLR inputs. Cell Rep. 2017;19(1):125-35.

42. Pena OM, Pistolic J, Raj D, Fjell CD, Hancock RE. Endotoxin tolerance represents a distinctive state of alternative polarization (M2) in human mononuclear cells. J Immunol. 2011;186(12):7243-54.

43. Julian MW, Strange HR, Ballinger MN, Hotchkiss RS, Papenfuss TL, Crouser ED. Tolerance and cross-tolerance following toll-like receptor (TLR)-4 and -9 activation are mediated by IRAK-M and modulated by IL-7 in Murine Splenocytes. PLoS One. 2015;10(7):e0132921.

44. Housley WJ, Fernandez SD, Vera K, Murikinati SR, Grutzendler J, Cuerdon N, Glick L, De Jager PL, Mitrovic M, Cotsapas C, et al. Genetic variants associated with autoimmunity drive NFKB signaling and responses to inflammatory stimuli. Sci Transl Med. 2015;7(291):291 ra293.

45. Miterski B, Bohringer S, Klein W, Sindern E, Haupts M, Schimrigk S, Epplen JT. Inhibitors in the NF[kappa]B cascade comprise prime candidate genes predisposing to multiple sclerosis, especially in selected combinations. Genes Immun. 2002;3(4):211-9.

\section{Submit your next manuscript to BioMed Central and we will help you at every step:}

- We accept pre-submission inquiries

- Our selector tool helps you to find the most relevant journal

- We provide round the clock customer support

- Convenient online submission

- Thorough peer review

- Inclusion in PubMed and all major indexing services

- Maximum visibility for your research

Submit your manuscript at www.biomedcentral.com/submit
Biomed Central 